\section{Salud, equidad y los Objetivos de Desarrollo del Milenio}

\section{Cristina Torres ${ }^{1}$ y Oscar J. Mújica ${ }^{1}$}

Palabras clave: formulación de políticas, evaluación de impacto, equidad en el acceso, indicadores de salud.

\footnotetext{
1 Área de Gobernanza y Política, Unidad de Políticas y Gobernanza (GPP/PG), Organización Panamericana de la Salud, Washington, D.C., EUA. La correspondencia debe dirigirse a Cristina Torres, Organización Panamericana de la Salud (GPP/PG), 525 Twenty-third Street, NW, Washington, DC 20037, Estados Unidos de América. Correo electrónico: torrescr@paho.org
}

En septiembre de 2000, representantes de 189 países se reunieron en la Cumbre del Milenio, convocada por las Naciones Unidas en la ciudad de Nueva York, y adoptaron la declaración que dio base a la formulación de los Objetivos de Desarrollo del Milenio (ODM). Estos objetivos forman parte de una larga serie de iniciativas emprendidas por los gobiernos, el sistema de las Naciones Unidas y las instituciones internacionales de financiamiento dirigidas a reducir la pobreza en el mundo. Por ello, para evaluar adecuadamente su importancia debe hacerse una lectura transversal con los resultados de otras conferencias de las Naciones Unidas, como la Cumbre Mundial sobre Medio Ambiente y Desarrollo, celebrada en Brasil en septiembre de 1992; la Conferencia Internacional sobre la Mujer, celebrada en Beijing, China, en septiembre de 1995; la Conferencia Internacional sobre Población y Desarrollo, realizada en El Cairo, Egipto, en septiembre de 1994; la Cumbre Mundial sobre Desarrollo Social, llevada a cabo en Copenhague, Dinamarca, en marzo de 1995; y la Conferencia Mundial contra el Racismo, la Discriminación Racial, la Xenofobia y las Formas Conexas de Intolerancia, celebrada en Durban, República Sudafricana, en agosto de 2001, entre otras. Estas conferencias también han servido para que en las agendas de los gobiernos se profundice en temas relacionados con los derechos sociales, el desarrollo sostenible y la protección del medio ambiente.

El resultado más visible de estas iniciativas que caracterizaron la década de 1990 fue, en primer lugar, el establecimiento de la voluntad política necesaria para buscar soluciones coordinadas entre los países desarrollados y los países en desarrollo, condición imprescindible para lograr el financiamiento que requiere su puesta en marcha. Otro importante resultado de estas iniciativas fue incorporar al proceso de negociación a las agencias técnicas de cooperación bilateral y multilateral como contrapartes de los organismos de financiamiento internacional. El tercer aporte de esta serie de reuniones cumbre de alcance mundial fue incorporar a la agenda internacional el tema de la pobreza, entendida esta como un obstáculo para el desarrollo que solo podría superarse en el marco de la llamada cooperación Norte-Sur. En otras rondas de negociaciones que tuvieron lugar durante la década de 1990 surgieron otras iniciativas con iguales propósitos, entre ellas la Iniciativa de Reducción de la Pobreza, de la cual forman parte en nuestra Región Bolivia, Guyana, Honduras y Nicaragua. 
Los ODM están vinculados con estas iniciativas previas y representan un avance por haberse logrado establecer ocho objetivos concretos: erradicar la pobreza y el hambre, lograr la universalización de la enseñanza primaria, promover la igualdad entre los sexos y la autonomía de la mujer, reducir la mortalidad infantil, mejorar la salud materna, combatir la infección por el virus de la inmunodeficiencia humana (VIH), el sida, el paludismo y otras enfermedades, garantizar la sostenibilidad del medio ambiente y fomentar una asociación mundial para el desarrollo. Además, se establecieron metas de desarrollo del milenio (MDM) e indicadores precisos para facilitar el seguimiento. Esto supera el perfil demasiado general y amplio de las propuestas emitidas en otras reuniones cumbre anteriores. Por último, se definió un horizonte temporal de 15 años, que es el tiempo necesario para lograr estos cambios, según los expertos.

La reacción internacional, particularmente la de las organizaciones del sistema de las Naciones Unidas y la de las entidades financieras internacionales, ha sido positiva. Como parte del sistema de las Naciones Unidas, la Organización Panamericana de la Salud (OPS) participa en el seguimiento de las ODM, tanto a escala nacional como regional, y le ha otorgado a esta iniciativa la más alta prioridad en su agenda de cooperación técnica para el quinquenio 2003-2007.

\section{LA DIMENSIÓN DE LA SALUD EN LOS ODM}

Varios temas relacionados con la salud se incorporaron a la Declaración de la Cumbre del Milenio. En el informe presentado por el Secretariado de la Organización Mundial de la Salud (OMS) en abril de 2003 se establece que "tres de los ocho objetivos, 8 de las 18 metas y 18 de los 48 indicadores están relacionados con la salud" (1). En el contenido de la Declaración se observa que, si bien las metas están vinculadas con la mejoría de la salud de las poblaciones en general, también hacen referencia a aspectos sanitarios puntuales - enfermedades de notificación obligatoria, acceso a los medicamentos, salud reproductiva, agua potable- con distintos niveles de responsabilidad sectorial.

Los tres objetivos identificados están encaminados explícitamente a reducir los riesgos relacionados con tres de los problemas de salud más acuciantes en las poblaciones vulnerables: la mortalidad materna, la mortalidad infantil y la infección por el VIH. El sector de la salud tendrá la responsabilidad de poner en marcha, monitorear y evaluar las medidas propuestas para alcanzar las metas formuladas. Las metas correspondientes a estos objetivos establecen la voluntad de "reducir en dos ter- cios la mortalidad en menores de 5 años entre 1990 y 2015" (meta 4); "reducir en tres cuartos la mortalidad materna entre 1990 y 2015" (meta 5); y "detener y revertir la epidemia del VIH para el 2015", así como "revertir la incidencia de malaria, tuberculosis y otras enfermedades prioritarias" (meta 6).

También compete al ámbito de la salud tratar de cumplir los objetivos asociados con otras dos metas: mejorar el acceso a los medicamentos esenciales (meta 8) y reducir la proporción de la población que no tiene acceso al agua potable (meta 7). En estos casos, el sector de la salud debe formar parte de equipos de trabajo más amplios, formados y coordinados por los ministerios y entidades encargados de la industria, el abastecimiento de agua, el saneamiento, etc.

Una vez identificados los ODM relacionados con el sector de la salud, cabe reflexionar acerca de la factibilidad de poder cumplirlos. En la Declaración del Milenio se establecen para cada indicador los valores que se deben alcanzar en un período de 15 años y la línea de base dada por los valores de esos indicadores en 1990. El diseño de las propuestas que formen parte del plan de acción y su puesta en marcha en los países insumirá, en el mejor de los casos, más de $20 \%$ del tiempo establecido ( 3 años).

Se debe destacar que los valores establecidos para cada uno de los indicadores están estrechamente ligados al conocimiento científico-técnico actual y los expertos coinciden en que no será posible alcanzar en todos los casos valores inferiores a los obtenidos ya en los países desarrollados.

Por otra parte, aunque las MDM establecen solo tasas nacionales, sin hacer referencia a la equidad, de su espíritu se desprende que la prioridad es mejorar las condiciones de vida de las personas en los segmentos más pobres de la población.

Los responsables de diseñar las políticas y programas de desarrollo se enfrentan al desafío de alcanzar los objetivos propuestos en los plazos establecidos, lo cual exigirá un respaldo político, la participación de la sociedad civil y la aplicación de un enfoque técnico dinámico. Algunas de las preguntas que surgen en este sentido son:

- ¿Será necesario realizar esfuerzos complementarios en América Latina y el Caribe o se alcanzarán los objetivos si se mantiene la tendencia actual?

- ¿Cómo se deben evaluar las MDM en el área de la salud: en términos de equidad, de vidas humanas o mediante el valor de los indicadores en cada país?

- ¿En qué países, subregiones o grupos poblacionales se debe concentrar el esfuerzo por alcanzar las MDM en materia de salud en América Latina y el Caribe? 
- ¿Cuáles son los instrumentos - tanto de las políticas como de los programas- más eficaces para avanzar en la consecución de las MDM desde el sector de la salud?

A fin de contribuir a este proceso, a continuación se analizan algunos elementos que pueden ayudar a responder estas interrogantes y que permitirán tomar decisiones mejor fundadas a escala nacional.

\section{¿Será posible alcanzar las metas de salud en la Región?}

La primera pregunta a la que dan lugar las discusiones previas a la toma de cualquier decisión bien fundada es si las políticas actuales en América Latina y el Caribe son suficientes para alcanzar estas metas o si se requieren cambios. Para responder a esta pregunta es necesario hacer un cuidadoso análisis de la información epidemiológica y socioeconómica existente.

Diversos problemas relacionados con la información estadística pueden hacer más difícil estimar la factibilidad de cumplir las metas propuestas y de realizar el seguimiento de todo el proceso. Primero, las fuentes de información estadística son variadas; ${ }^{2}$ segundo, no están compatibilizadas; y por último, no tienen el nivel de desagregación necesario para poder diseñar y hacer el seguimiento de las políticas orientadas a reducir la pobreza. El Programa de las Naciones Unidas para el Desarrollo (PNUD), por ejemplo, concluye a partir del análisis de sus fuentes estadísticas que la subregión de América Latina y el Caribe no tendría que poner en marcha medidas correctoras para lograr las metas propuestas en los plazos acordados. En su Informe de Desarrollo Humano 2003 (2) se expone que la tasa de mortalidad en menores de 5 años en América Latina y el Caribe bajó de 56 por mil nacidos vivos en 1990 a 35 por mil nacidos vivos en 2001 y que, de mantenerse la tendencia actual, se puede superar la meta propuesta para el año 2015.

Sin embargo, otras agencias, a partir del análisis de los datos de otras fuentes, no comparten ese optimismo. Estudios recientes de la Comisión Económica para América Latina y el Caribe (CEPAL) (3), realizados mediante modelos de simulación en 18 países de América Latina y el Caribe, concluyeron que si no se modifican las políticas actuales, solo 7 de los 18 países estudiados podrían alcanzar los

\footnotetext{
2 Existe una multiplicidad de fuentes: de diversas agencias de las Naciones Unidas (como UNICEF, PNUD, OPS, OMS, División de Población, División de Estadísticas), de organismos financieros (Banco Mundial, Banco Interamericano de Desarrollo) y de instituciones nacionales (ministerios de salud, oficinas nacionales de estadísticas y otras).
}

FIGURA 1. Trayectorias posibles de la mortalidad en menores de 5 años según dos escenarios. América Latina y el Caribe, 1990-2015

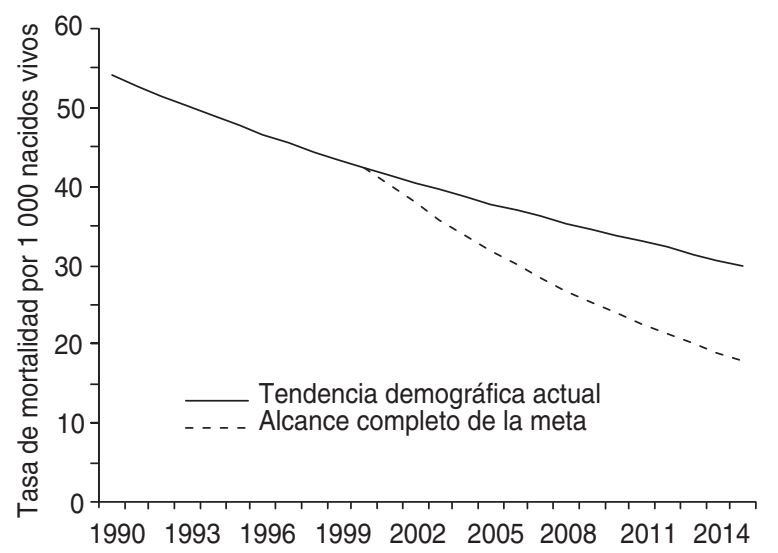

objetivos asociados con la reducción de la pobreza ${ }^{3}$ para 2015 (Argentina, ${ }^{4}$ Chile, Colombia, Honduras, Panamá, República Dominicana y Uruguay). En un segundo grupo de seis países se reduciría la incidencia de la extrema pobreza, pero a un ritmo muy lento (Brasil, Costa Rica, El Salvador, Guatemala, México y Nicaragua). Los otros cinco países (Bolivia, Ecuador, Paraguay, Perú y Venezuela) experimentarían un aumento en los niveles de extrema pobreza debido al aumento de la inequidad, al descenso del ingreso per cápita o a ambos.

Por su parte, la OPS, ${ }^{5}$ con datos de la División de Población de las Naciones Unidas, ha calculado que de mantenerse la tendencia observada en la mortalidad de menores de 5 años entre 1990 y 2001 ( 54,5 y 41,4 por mil nacidos vivos, respectivamente), el valor de este indicador podría llegar a 30,0 por mil nacidos vivos en el año 2015, prácticamente el doble de lo propuesto por las MDM, que consiste en reducirlo a 18 por mil nacidos vivos (figura 1). En resumen, si no se aplican medidas correctoras en las políticas actuales es posible que no se cumplan las predicciones optimistas del PNUD y que no se alcancen las metas propuestas para el año 2015.

De lo expuesto se desprende claramente la importancia de armonizar las diferentes bases de

\footnotetext{
3 Existen diferentes metodologías para calcular los índices de pobreza Las más conocidas son la de la CEPAL y la del Banco Mundial. Para más información acerca de este tema, puede verse el debate sobre la definición de pobreza y una perspectiva comparativa ofrecida por Ruggeri, Saith y Stewart (4), y Feres y Mancero (5). Para una revisión de los indicadores sociales y las bases estadísticas empleadas para calcular las metas, véase el trabajo de Gutiérrez Ezpeleta (6).

4 Este estudio se basó en los datos anteriores a la crisis económica y política que vivió Argentina en 2002.

5 Estas estimaciones se obtuvieron para este trabajo mediante la unidad de Políticas y Gobernanza de la Organización Panamericana de la Salud.
} 
datos estadísticos de salud pertenecientes al PNUD, a la División de Población de las Naciones Unidas, al Fondo de las Naciones Unidas para la Infancia (UNICEF), a la OPS y a los distintos países. De esta forma se logrará contar con un instrumento eficaz para la toma de decisiones y el seguimiento de este proceso.

\section{¿Pueden las MDM contribuir a reducir las inequidades de salud en la Región?}

América Latina y el Caribe es la región que presenta mayores inequidades, por lo que se impone hacer un análisis complementario que, además de ayudar a elevar los valores medios nacionales de los indicadores, profundice en los elementos asociados con la distribución de los factores que determinan el estado de salud de la población.

Como las MDM son parte de una estrategia dirigida a mejorar la situación de los grupos más vulnerables, es necesario reflexionar acerca de qué segmentos de la población están más expuestos a la pobreza y la exclusión, a fin de redirigir los esfuerzos adecuadamente. A pesar de que en el texto de las MDM no se menciona explícitamente, la puesta en marcha de las estrategias en América Latina y el Caribe se vería enriquecida si se añadiesen algunas acciones dirigidas a mejorar el acceso a los servicios de los grupos más vulnerables, dada la gran inequidad observada en la Región.

Para lograr este propósito es necesario identificar adecuadamente las poblaciones objetivo. Según distintas fuentes, una de las nuevas características de las poblaciones afectadas por la pobreza es que se han trasladado de las zonas rurales a las ciudades. ${ }^{6}$ A pesar de que la pobreza es más crítica en zonas rurales, en estas hay menos hogares y una menor población. En América Latina, 60\% de los pobres viven en zonas urbanas y esto ha contribuido al elevado nivel de indigencia que reina en sus ciudades.

Otra característica actual de la pobreza es el cambio que ha sufrido el perfil poblacional de los grupos excluidos: por un lado, la pobreza se ha fe-

\footnotetext{
6 Publicaciones recientes señalan entre las causas de la pobreza la depauperación derivada del desempleo, las dificultades en la transferencia de tecnologías, los cambios en el mercado de trabajo (particularmente, la flexibilización laboral), la inflación y las privatizaciones. Todo esto conduce al deterioro de los ingresos, a la reducción del número de empleos públicos derivados de la reforma del Estado - que afecta a las clases medias urbanas-, a la inaccesibilidad a la compra de servicios y a la pérdida de beneficios sociales debido al aumento de puestos de trabajo no formalizados. Estas causas confluyen en la conformación de la "nueva pobreza". Kessler G, Di Virgilio M. Perspectives on urban poverty in Latin America. La nueva pobreza urbana en Argentina y América Latina. Washington, D.C.: Woodrow Wilson Center of Public Policy; 2003 (documento de trabajo).
}

minizado y los hogares encabezados por mujeres son los más afectados. También ha aumentado el porcentaje de niños y jóvenes pobres, y el desempleo afecta en mayor medida a los que buscan trabajo por primera vez sin tener experiencia laboral. Por último, los hogares integrados por una sola persona, por lo general un adulto mayor, también son más vulnerables que los formados por núcleos familiares más numerosos, donde las estrategias para la supervivencia se diversifican y aumenta el capital social. ${ }^{6}$

A estas nuevas categorías que conforman la "nueva pobreza" debe añadirse un enfoque étnico que permita identificar a otros grupos que siempre han estado excluidos de los beneficios sociales. Tal es el caso de los pueblos indígenas y de las comunidades de descendientes de africanos, que presentan inequidades insoslayables en sus condiciones sanitarias y de vida, así como en el acceso a los servicios de salud y de educación. Un estudio realizado en 2001 por el Banco Interamericano de Desarrollo (BID) revela que en Perú, $80 \%$ de la población indígena era pobre mientras que $50 \%$ de la población no indígena se situaba por debajo de la línea de la pobreza. En el caso de México, la brecha es aun mayor, pues más de $80 \%$ de la población indígena se encuentra por debajo del umbral de la pobreza, mientras que solo $18 \%$ de la población en general comparte esa situación (7). Según la CEPAL, situaciones similares se pueden encontrar en Bolivia, Guatemala y Paraguay (8).

Por razones históricas semejantes, los descendientes de africanos, otro grupo étnico muy numeroso, ${ }^{7}$ presentan también una situación de alta vulnerabilidad. Según un análisis de los datos de la Pesquisa Nacional por Amostreo Domiciliar (PNAD) realizada en 1996 en Brasil, las minorías étnicas están mucho más representadas en los grupos de ingresos más bajos, de manera que $27,88 \%$ de las personas ubicadas en el quinto inferior en términos de ingresos son indígenas, $52,5 \%$ son descendientes de africanos y solo 13,37\% son descendientes de europeos (9).

Las diferencias en los ingresos según el grupo étnico no son una característica exclusiva de los países en desarrollo, pues también se observan en los países desarrollados de la Región, entre ellos los Estados Unidos. Según los datos de la Oficina del Censo de ese país, el ingreso promedio por habitante en 1999 era de US\$ 14397 para los estadounidenses descendientes de africanos, de \$11 621 para los de origen hispano y de $\$ 24109$ para los blancos (10).

\footnotetext{
7 Según cálculos, los descendientes de africanos ascienden a unos 150 millones de personas, de los cuales Brasil y Estados Unidos tienen las comunidades más numerosas.
} 
Por lo tanto, el desafío consiste en diseñar programas e instrumentos de políticas que permitan alcanzar las metas para el año 2015 teniendo en cuenta las características de la pobreza en América Latina y el Caribe. En este sentido, hay tres posibles estrategias en lo que respecta a las políticas: 1) no realizar cambio alguno, 2) desarrollar una estrategia por "derrame" ${ }^{8}$ y 3) aplicar una estrategia enfocada en los grupos de población más desfavorecidos (minorías étnicas, mujeres jefas de hogar, poblaciones urbanas periféricas) y con mayores necesidades sanitarias.

Las estrategias dirigidas a los grupos de más bajos recursos podrían llevar a alcanzar las metas de salud y generarían beneficios complementarios en términos de equidad distributiva (11).

\section{ESTUDIO DE CASO: LA MORTALIDAD INFANTIL EN BRASIL}

Con el objetivo de explorar el impacto sobre la mortalidad infantil ${ }^{9}$ atribuible a la aplicación de intervenciones basadas en las tres estrategias de política indicadas, se preparó un estudio de caso con la información oficial disponible de Brasil.

En 1990, la mortalidad en menores de un año en Brasil fue de 49,4 por mil nacidos vivos; por lo tanto, para reducir este indicador en dos tercios, la mortalidad de este grupo debe reducirse en todo el país a 16,5 por mil nacidos vivos en el año 2015. En el año 1999, este indicador disminuyó a 36,2 por mil nacidos vivos; si la tendencia decreciente observada entre 1990 y 1999 se mantuviera con el mismo ritmo de reducción se podría esperar alcanzar una tasa de mortalidad infantil de 32,9 por mil nacidos vivos, por lo que no se alcanzaría la meta propuesta (figura 2). De hecho, en términos de carga de mortalidad, entre 1999 y 2015 se evitarían 200 mil muertes infantiles de las $700 \mathrm{mil}$ (la "población objetivo") requeridas para cumplir plenamente esa meta. ${ }^{10}$ Esto significa que si no se aplican cambios en las políticas vigentes se mantendrían las tendencias demográficas actuales y el país alcanzaría solamente $27 \%$ de lo propuesto en la meta 4 (figura 3).

\footnotetext{
8 Se alude aquí a la estrategia del "trickle-down", que hace hincapié en las políticas tendientes a la reactivación económica como estrategia para la incorporación progresiva de sectores de la población al desarrollo humano.

9 Debido a su relevancia sanitaria, para este ejercicio analítico se tomó la mortalidad infantil (menores de 1 año) y no la de menores de 5 años, como indica la meta 4 de los MDG. La validez del enfoque, no obstante, se mantiene intacta; de hecho, en las condiciones epidemiológicas actuales, alcanzar la meta 4 implicaría reducir la mortalidad infantil aproximadamente entre 58 y $83 \%$.

10 Si la tasa de mortalidad infantil observada en 1999 se mantuviera constante entre 2000 y 2015, en Brasil se acumularían 2,14 millones de muertes en menores de 1 año de edad; este es el llamado pool de incidencia (de mortalidad infantil).
}

FIGURA 2. Trayectorias posibles de la mortalidad en menores de 5 años según dos escenarios. Brasil, 1990-2015

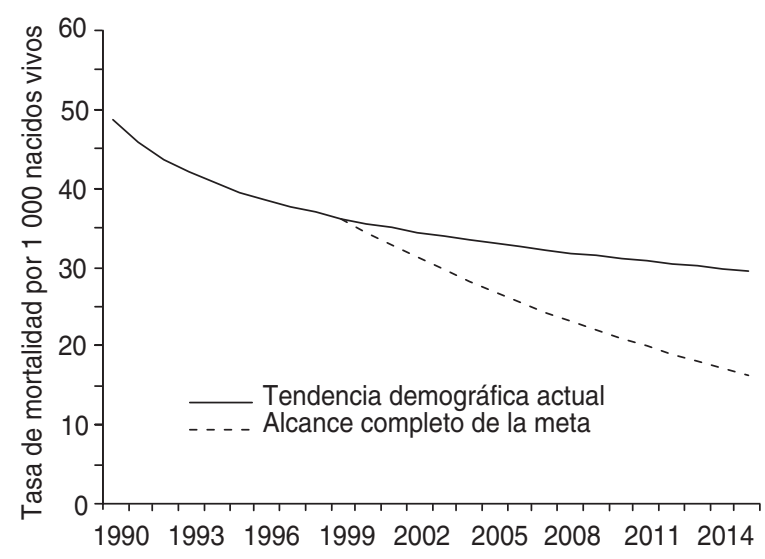

Esto indica que se necesita realizar un esfuerzo adicional -mediante políticas, estrategias e intervenciones- para reducir la mortalidad en menores de un año hasta la cifra establecida en las MDM. Es posible construir varios escenarios para calcular el impacto de las distintas estrategias en términos de lograr la meta propuesta y de reducir las brechas existentes entre los diferentes estratos socioeconómicos de la población. Para este análisis se aprovechó la existencia de dos estratos definidos "por corte natural" en la población brasileña y que se diferencian marcadamente entre sí. El primero, estrato A, está compuesto por la población de cuatro estados del sur de Brasil (Paraná, Rio Grande do Sul, Santa Catarina y São Paulo) y concentra el $80 \%$ de la población blanca, urbana, que sabe leer y escribir y que tiene altos ingresos; el estrato B está conformado por la población de los otros 23 estados, en los cuales se concentra $80 \%$ de la población descendiente de africanos, rural, analfabeta y de bajos ingresos.

Según estos escenarios y estratos, se aplicó un modelo de simulación para cuantificar el efecto preventivo - expresado en número de muertes infantiles evitadas y la proporción de éxito con respecto a la meta $4-\mathrm{y}$ el impacto potencial en la salud pública - expresado tanto mediante la reducción del riesgo de muerte infantil (tasas de mortalidad infantil) como mediante la magnitud de la brecha media de este riesgo entre ambos estratos (riesgo relativo).

Para este análisis se utilizaron diferentes opciones de intervención dirigidas a reducir la mortalidad infantil en la población de ambos estratos según distintos grados o coberturas de "focalización" de los esfuerzos de prevención de la mortalidad infantil en ambos estratos. Estos esfuerzos están referidos tanto a la "población objetivo" (es 
FIGURA 3. Carga de mortalidad infantil evitada según dos escenarios. Brasil, 1990-2015

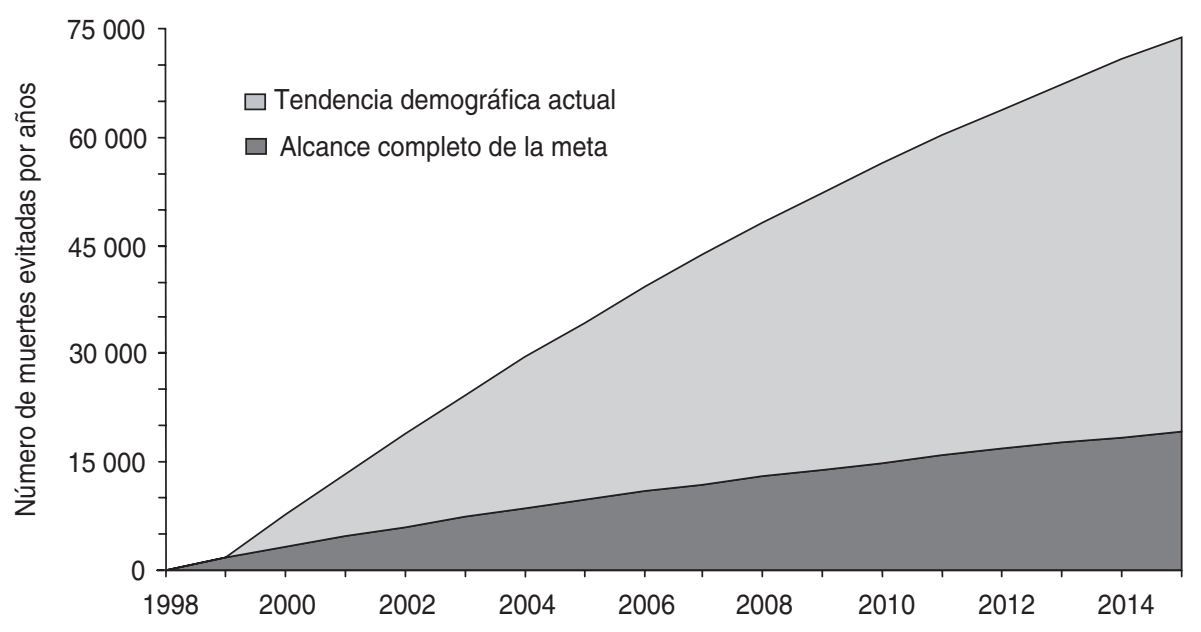

decir, a la parte de las 700 mil muertes evitables correspondientes a cada estrato) como al pool de incidencia (es decir, al total de muertes infantiles acumuladas en el período 2000-2015 que podrían evitarse). Este ejercicio representa solamente una aproximación exploratoria al objetivo principal, que consiste en definir políticas socialmente eficientes para elevar al máximo la distribución poblacional de las ganancias potenciales que resultaría de cumplir las MDM (cuadro 1).

Deben destacarse las diferencias en el impacto derivado de la aplicación de dos estrategias contrapuestas. Si se adoptara una estrategia "por derrame" - es decir, aquella que se basa en acciones preventivas dirigidas a los grupos poblacionales que presentan mejores indicadores de salud (o sea, focalizada en el estrato A) - con intervenciones, por ejemplo, orientadas a evitar el $90 \%$ del total de las muertes infantiles evitables en los cuatro estados más ricos de Brasil, dejando a la población del estrato B bajo la tendencia decreciente actual (o sea, en caída monotónica), se lograría alcanzar el $80 \%$ de la meta 4 en el año 2015, pero la brecha media entre ambos estratos se ampliaría de 1,7 a 27,0 , es decir, la desigualdad se incrementaría en más de 15 veces (cuadro 1 ).

Por el contrario, si se adopta una estrategia focalizada en la búsqueda de la equidad - concentrada en la población con mayores necesidades de salud y más vulnerable (es decir, en el estrato B)con acciones dirigidas, por ejemplo, a reducir el $40 \%$ del total de muertes infantiles en ese estrato, mientras el estrato A queda bajo la tendencia decreciente actual, se podría alcanzar el $97 \%$ de la meta 4 $\mathrm{y}$, además, se conseguiría revertir la inequidad existente entre ambos estratos en relación con el riesgo de muerte infantil, ya que se eliminaría el exceso de riesgo de muerte infantil que se observa actualmente en el estrato B (cuadro 1).

El modelo de simulación sugiere la existencia de una transacción electiva o trade-off entre la eficiencia con que se puede alcanzar la meta 4 de las MDM y la equidad con la que se pretende lograr esa meta $-\mathrm{y}$ ello se aplica también a las otras MDM asociadas con la salud. Solo se podrá alcanzar el objetivo propuesto en la meta 4 si se aplican acciones para la prevención de la mortalidad infantil. Sin embargo, la aplicación de una estrategia trickle-down o "por derrame" ayudaría a acercarse a la meta sin modificar significativamente la brecha existente en cuanto a la mortalidad infantil entre las poblaciones de diferentes estratos socioeconómicos. Para alcanzar la meta 4 con equidad es necesario realizar un esfuerzo adicional para disminuir el número de muertes infantiles en las poblaciones con mayor grado de exclusión social. Este estudio de caso demuestra que se puede alcanzar la meta 4 de las MDM y lograr que las tasas de mortalidad infantil sean similares en ambos estratos en el año 2015.

\section{Conclusiones del estudio de caso}

Las opciones presentadas en este análisis, con sus correspondientes escenarios, solo constituyen un ejemplo de cómo se pueden adoptar los lineamientos de políticas en materia de salud y cuáles son los elementos que se deben tener en consideración para tomar decisiones que tengan un mayor impacto en términos de equidad y de protección de los grupos más vulnerables.

A modo de síntesis, de la información que ofrece este estudio de caso pueden destacarse las siguientes conclusiones: 
CUADRO 1. Impacto de diversas estrategias de focalización en la mortalidad infantila y la desigualdad de estratos socioeconómicos diferentes. Brasil, 2000-2015

\begin{tabular}{|c|c|c|c|c|c|c|c|c|c|}
\hline \multicolumn{2}{|c|}{ Estrategia } & \multicolumn{3}{|c|}{ Efecto preventivo } & \multirow{3}{*}{$\begin{array}{l}\text { Proporción } \\
\text { de éxito } \\
(\%)\end{array}$} & \multicolumn{4}{|c|}{ Impacto en salud pública } \\
\hline \multicolumn{2}{|c|}{ Focalización (cobertura) } & \multicolumn{3}{|c|}{ Muertes infantiles evitadas } & & \multicolumn{3}{|c|}{ Tasa-resumen mortalidad infantil } & \multirow{2}{*}{$\begin{array}{c}\text { Brecha } \\
\text { media } \\
\text { B/A }\end{array}$} \\
\hline Estrato A & Estrato B & Estrato $\mathrm{A}$ & Estrato B & Brasil & & Estrato A & Estrato $\mathrm{B}$ & Brasil & \\
\hline \multicolumn{10}{|c|}{$\begin{array}{l}\text { Sobre la fracción preventiva } \\
\text { (población objetivo) }\end{array}$} \\
\hline Caída monotónica & Caída monotónica & 39682 & 153697 & 193379 & 27,4 & 20,8 & 36,0 & 29,6 & 1,73 \\
\hline $90 \%$ & Caída monotónica & 102482 & 153697 & 256179 & 42,0 & 11,2 & 36,0 & 26,8 & 3,21 \\
\hline $80 \%$ & Caída monotónica & 91095 & 153697 & 244792 & 40,4 & 12,4 & 36,0 & 27,2 & 2,91 \\
\hline $70 \%$ & Caída monotónica & 79708 & 153697 & 233406 & 38,7 & 13,6 & 36,0 & 27,6 & 2,64 \\
\hline $80 \%$ & $20 \%$ & 91095 & 79551 & 170646 & 51,6 & 12,4 & 35,9 & 27,3 & 2,90 \\
\hline $60 \%$ & $40 \%$ & 68321 & 159102 & 227423 & 59,7 & 14,9 & 30,9 & 25,3 & 2,07 \\
\hline $50 \%$ & $50 \%$ & 56934 & 198877 & 255812 & 63,7 & 16,2 & 28,5 & 24,3 & 1,76 \\
\hline $40 \%$ & $60 \%$ & 45548 & 238653 & 284200 & 67,7 & 17,6 & 26,2 & 23,4 & 1,49 \\
\hline $20 \%$ & $80 \%$ & 22774 & 318203 & 340977 & 75,8 & 20,5 & 21,9 & 21,5 & 1,06 \\
\hline $40 \%$ & $80 \%$ & 45548 & 318203 & 363751 & 79,0 & 17,6 & 21,9 & 20,8 & 1,24 \\
\hline $60 \%$ & $80 \%$ & 68321 & 318203 & 386525 & 82,3 & 14,9 & 21,9 & 20,1 & 1,47 \\
\hline $80 \%$ & $80 \%$ & 91095 & 318203 & 409299 & 85,5 & 12,4 & 21,9 & 19,4 & 1,77 \\
\hline Caída monotónica & $70 \%$ & 39682 & 278428 & 318110 & 66,9 & 20,8 & 24,0 & 22,8 & 1,15 \\
\hline Caída monotónica & $80 \%$ & 39682 & 318203 & 357885 & 72,6 & 20,8 & 21,9 & 21,6 & 1,05 \\
\hline Caída monotónica & $90 \%$ & 39682 & 357979 & 397661 & 78,2 & 20,8 & 19,8 & 20,3 & 0,95 \\
\hline $100 \%$ & $100 \%$ & 113869 & 397754 & 511623 & 100,0 & 10,1 & 17,9 & 16,5 & 1,78 \\
\hline \multicolumn{10}{|c|}{$\begin{array}{l}\text { Sobre el pool de incidencia } \\
\text { (sobre la meta) }\end{array}$} \\
\hline $50 \%$ & Caída monotónica & 229123 & 153697 & 382820 & 54,3 & 6,7 & 36,0 & 24,7 & 5,37 \\
\hline $70 \%$ & Caída monotónica & 320772 & 153697 & 474469 & 67,3 & 4,0 & 36,0 & 23,4 & 8,96 \\
\hline $90 \%$ & Caída monotónica & 412421 & 153697 & 566119 & 80,3 & 1,3 & 36,0 & 22,0 & 26,87 \\
\hline Caída monotónica & $40 \%$ & 39682 & 643906 & 683588 & 97,0 & 20,8 & 14,2 & 16,2 & 0,68 \\
\hline Caída monotónica & $50 \%$ & 39682 & 804883 & 844565 & 119,8 & 20,8 & 11,9 & 14,3 & 0,57 \\
\hline Caída monotónica & $60 \%$ & 39682 & 965860 & 1005541 & 142,6 & 20,8 & 9,5 & 12,4 & 0,46 \\
\hline
\end{tabular}

Fuente: Unidad de Políticas y Gobernanza, Organización Panamericana de la Salud, 2003.

a De menores de un año.

- Las formas de posicionarse frente a las metas son variadas y dependerán de decisiones políticas, no solamente técnicas.

- Los resultados finales de las acciones que se realicen para alcanzar las MDM no se miden únicamente mediante los valores promedio de la tasa de mortalidad infantil, sino también en términos del número de vidas salvadas y del grado de equidad logrado, según la magnitud de la brecha que existe entre los más ricos y los más pobres.

- La comparación del impacto de dos de las estrategias de intervención posibles (opciones 2 y 3) es contrastante: en el primer escenario (opción 2), los esfuerzos estarían orientados a evitar $90 \%$ del total de las muertes infantiles evitables en los cuatro estados más ricos de Brasil, y dejan al resto bajo la tendencia decreciente actual. En tales condiciones, en el año 2015 se lograría alcanzar 80\% de la meta 4 de las MDM, pero la brecha entre ambos estratos se incrementaría en más de 15 veces. Por otra parte, si se adoptara una estrategia enfocada en los grupos más vulnerables —concentrada en los estados que presentan condiciones de mayor exclusión social (opción 3) - se obtendría una reducción de $40 \%$ del total de muertes infantiles en el estrato B, mientras que el resto seguiría mostrando la tendencia decreciente actual, con lo que se alcanzaría la meta propuesta y, además, una mayor equidad.

- El no realizar esfuerzos complementarios es la peor opción en términos de muertes evitadas y de la posibilidad de cumplir la meta propuesta.

- En la realidad, será posible encontrar combinaciones más moduladas, que tomen en cuenta los esfuerzos realizados en los estados más desarrollados y las iniciativas que se apliquen en las regiones con las peores condiciones, según un diseño que busque focalizar los beneficiarios. La combinación de diferentes estrategias será sin duda lo que permitirá alcanzar resultados satisfactorios, tanto social como políticamente.

\section{¿CÓMO DEFINIR ADECUADAMENTE LOS BENEFICIARIOS?}

Como se ha visto, alcanzar las MDM no es un mero ejercicio mecánico, sino un proceso que requiere de una reingeniería de las políticas dirigidas a reducir la inequidad en materia de salud en la Re- 
gión. Se deben rediseñar las políticas y los programas de salud en función de los grupos vulnerables, centrarse en las regiones menos favorecidas y hacer hincapié en los esfuerzos aplicados en los países con índices de desarrollo menos favorables.

Las prioridades para localizar los esfuerzos deberán definirse según dos criterios que pueden usarse por separado o simultáneamente, según el caso. El primer criterio consiste en identificar los países prioritarios, es decir, los países cuyos indicadores de pobreza presenten valores definidos como de alta vulnerabilidad y donde aumentará el nivel de pobreza en 2015 si no se aplica una intervención vigorosa. A partir de esta información $-\mathrm{y}$ tomando en cuenta los países que son de prioridad para la OPS, la Iniciativa de Reducción de la Pobreza y la CEPAL - se puede definir que los países que requieren especial atención a la luz de sus indicadores socioeconómicos son Bolivia, Haití, Guyana, Honduras, Nicaragua, Ecuador, Paraguay, Perú y Venezuela.

El segundo criterio está relacionado con los segmentos de población excluidos debido a su situación geográfica, origen étnico, sexo o condición socioeconómica. Según este criterio, no solo se debe trabajar en los países mencionados más arriba, sino también en otros cuyos índices de pobreza pueden ser menores, pero en los que existen poblaciones excluidas (regiones, grupos étnicos o raciales, grupos marginales urbanos, zonas fronterizas) que presentan valores muy inferiores a la media de sus respectivos países y donde es necesario hacer un esfuerzo particular para mejorar sus indicadores. Tal es el caso, por ejemplo, de la costa del Pacífico de Colombia, la costa atlántica de Honduras y el nordeste brasileño, donde habitan grupos étnicos marginados. Con frecuencia, estas regiones y grupos no están ubicados en un solo país, por lo que se hace imprescindible coordinar las acciones entre dos países o más.

\section{Modelos de intervención}

El modelo de intervención debe tener en cuenta cuáles son las acciones más eficientes y eficaces y cuál es la perspectiva o la estrategia más general en la que se insertará esa intervención. En relación con las intervenciones mismas, existe un gran acopio de capacidad técnica en las áreas de desarrollo donde se centran las MDM. ${ }^{11}$

\footnotetext{
11 Estos temas han sido objeto de revisión por los cuerpos directivos en innumerables ocasiones. Se han adoptado más de 30 resoluciones entre 1993 y 2002. Véase: Torres C, Singleton M. Resoluciones sobre los temas de MDG 1993-2002. Washington, D.C.: Organización Panamericana de la Salud; 2003 (documento interno de trabajo).
}

Por lo tanto, para definir el modelo de intervención es crucial volver sobre la discusión de cuál ha de ser la estrategia global. No hay consenso acerca de si se debe aplicar la estrategia universalista o la de focalización. "La acusación predominante que se suele hacer a las políticas sociales en América Latina es que las mismas se apoyaron en un modelo de incorporación corporativo con un sesgo urbano y altamente estratificado; en la medida en que se expandían estos sistemas se incrementaban los niveles de desigualdad en el interior de los programas favoreciendo a los sectores más influyentes [...] y también crecían sus burocracias, las que se apropian de una mayor proporción del gasto social, disminuyendo el beneficio neto que alcanzaba a los destinatarios originales de los programas."12

"El universalismo ha sido parte del discurso tradicional de los gobiernos latinoamericanos [...], pero la práctica latinoamericana siempre mostró desigualdades notorias en la provisión, por lo que se ha hablado de universalismo 'excluyente', de casos 'estratificados' [...]. Para superar las diferencias existentes, es necesario tratar desigualmente a quienes son social y económicamente desiguales, mediante la acción afirmativa o la discriminación positiva" (12).

Por supuesto, en los países de la Región coexisten otras formas de ver el "universalismo" que probablemente le otorgan características positivas en cuanto a la construcción de una sociedad más integrada o democrática. Aun sin profundizar, es importante señalar que se pueden combinar instrumentos focalizados y acciones afirmativas con otros elementos de carácter igualitario, a fin de garantizar la distribución de beneficios al conjunto más amplio de la población de cada país. Es importante señalar la distinción entre el universalismo como resultado de una política y el universalismo como categoría para la selección de los beneficiarios en el diseño de los programas. Es posible que para aproximarse al universalismo en sus resultados una política deba incorporar acciones afirmativas (13) o programas focalizados como forma de revertir las inequidades que se han generado por razones políticas y económicas. Sin embargo, tratar de forma desigual a los desfavorecidos puede ser una estrategia transitoria que podría dar lugar a programas universales para la identificación de los beneficiarios una vez que se haya logrado la igualdad en las condiciones iniciales.

Por último, en los países donde la población que vive por debajo de la línea de pobreza supera el $40 \%$ de la población total, no siempre es posible

\footnotetext{
12 Filgueira C. La pobreza y el desarrollo social en las agendas globales, regionales y nacionales. Montevideo: Organización de Estados Americanos; 1997 (documento de trabajo).
} 
seleccionar programas focalizados para enfrentar los problemas de desigualdad. Estos programas se aplican mejor y se obtienen resultados más satisfactorios en países donde los segmentos con mayores carencias representan menos de $20 \%$ de la población y donde - ya sea por razones de localización geográfica, de origen étnico, de edad o de sexo- la identificación de los beneficiarios es relativamente sencilla.

Es necesario tener siempre en cuenta las estrategias generales, como el fortalecimiento de la atención primaria de salud, que han sido aplicadas en la Región durante 25 años y cuya evaluación es muy positiva por facilitar el acceso y promover la participación de los diversos niveles del sector público y privado y de las comunidades (14).

\section{PRINCIPALES DESAFÍOS}

Los principales desafíos para poder cumplir las MDM son:

- Mejorar y armonizar los sistemas de información de salud como prioridad, ya que de lo contrario no se podrán identificar adecuadamente las poblaciones objetivo ni se podrá afinar la selección de beneficiarios para los programas. Esto implica que los programas podrían desviarse y beneficiar a otros segmentos poblacionales, con la consiguiente pérdida de impacto.

- Diseñar programas de salud vinculados con los ODM que articulen adecuadamente el conjunto de prestaciones e intervenciones de mayor impacto, según las características propias de las poblaciones a quienes se quiere beneficiar. Para ello se deben tomar en cuenta su ubicación geográfica, su condición socioeconómica y su origen étnico, factores que muchas veces constituyen barreras para la eficaz puesta en marcha de los programas de salud. Se recomienda llevar a cabo un diálogo permanente entre la comunidad y los encargados de desarrollar los programas.

- Fortalecer la voluntad política de apoyar los ODM. Las políticas de salud orientadas a aliviar la pobreza requieren de una voluntad explícita de los gobiernos de apoyar los cambios que estas implican y de garantizar el financiamiento necesario. A partir del año 2003 y hasta el 2005, están programadas elecciones presidenciales en Chile, Honduras, México, Guatemala, El Salvador, Estados Unidos, Panamá, República Dominicana y Uruguay. Después del año 2005 habrá nuevas elecciones en estos y en otros países. Se calcula que en el período 2006-2015 ocurrirán 42 cambios de administración, por lo que se recomienda trabajar en las escalas regional y nacional en la cre- ación de consensos que permitan lograr una mayor estabilidad (mantenimiento de equipos de trabajo, financiamiento y visibilidad política), la cual frecuentemente se ve afectada durante los cambios de gobierno. Asimismo, se debe prever la adopción de políticas de estado que garanticen la continuidad de los procesos hasta su culminación en 2015.

- Se debe garantizar el financiamiento de las acciones emprendidas para cumplir los ODM. Tomando en consideración que existen situaciones nacionales que no permiten - por lo menos en la etapa inicial - cubrir la inversión de la puesta en marcha de programas y políticas con fondos del presupuesto nacional, se recomienda la formulación de proyectos para acceder a fondos internacionales. Esta tarea requerirá la capacitación de equipos de los ministerios de salud a nivel nacional en el diseño de proyectos para llevar a cabo eficazmente las negociaciones con agencias de cooperación internacional e instituciones financieras.

No cabe duda de que los ODM constituyen una oportunidad para crear las condiciones que permitan a millones de habitantes de la Región mejorar sus condiciones de vida y alcanzar una mayor equidad en el acceso a los servicios. El esfuerzo que deben realizar los gobiernos para alcanzar los indicadores propuestos no debe de ninguna manera desviarse de su verdadero objetivo final de eliminar la desigualdad que sufren segmentos importantes de la población. En palabras de Kofi Annan, Secretario General de las Naciones Unidas, dirigidas a los Jefes de Estado reunidos en la Cumbre Iberoamericana en 2003, "algunos grupos sufren en grado desproporcionado. Se sigue marginando a las personas de ascendencia africana, al igual que a los pueblos indígenas, ya se trate de mayorías o de minorías [...]. Una estrategia de desarrollo digna de ese nombre no puede soslayar las desigualdades patentes entre los distintos grupos sociales, lo cual es aún más cierto cuando las diferencias socioeconómicas coinciden con las divisiones étnicas" (15).

\section{SYNOPSIS}

\section{Health, equity, and the Millennium Development Goals}

In September 2000 representatives of 189 countries met for the Millennium Summit, which the United Nations convened in New York City, and adopted the declaration that provided the basis for formulating the Millennium Development Goals (MDGs). The eight goals are part of a long series of initiatives that governments, the United Nations 
system, and international financial institutions have undertaken to reduce world poverty. Three of the eight goals deal with health, so the health sector will be responsible for implementing, monitoring, and evaluating measures proposed to meet targets that have been formulated: to reduce by twothirds the mortality rate in children under 5 years of age between 1990 and 2015; to reduce by three-quarters the maternal mortality rate between 1990 and 2015; and to halt and begin to reverse the spread of HIV/AIDS by the year 2015, as well as to halt and begin to reverse the incidence of malaria, tuberculosis, and other major diseases. The health sector must also work with other parties to achieve targets connected with two other of the goals: to improve access to affordable essential drugs, and to reduce the proportion of persons who do not have safe drinking water. Adopting a strategy focused on the most vulnerable groups-ones concentrated in locations and populations with the greatest social exclusion-would make possible the largest total reduction in deaths among children, thus reaching the proposed target as well as producing greater equity. In the Region of the Americas the principal challenges in meeting the MDGs are: improving and harmonizing health information systems; designing health programs related to the MDGs that bring together the set of services and interventions that have the greatest impact, according to the special characteristics of the populations who are intended to be the beneficiaries; strengthening the political will to support the MDGs; and guaranteeing funding for the measures undertaken to attain the MDGs.

\section{REFERENCIAS}

1. Organización Mundial de la Salud. La contribución para el logro de las Metas de Desarrollo de la Declaración del Milenio de Naciones Unidas. Informe del Secretariado. Ginebra: OMS; 2003.

2. United Nations Development Program Human Development Report 2003. Millennium Development Goals: A compact among nations to end human poverty. New York: UNDP; 2003.

3. Comisión Económica para América Latina y el Caribe. Situación y perspectivas 2003. Estudio económico de América Latina y el Caribe 2002-2003. Santiago, Chile: Naciones Unidas, CEPAL; 2003. Hallado en: http://www.eclac.cl/cgibin/getProd.asp? $x \mathrm{xml}=/$ publicaciones/ $\mathrm{xml} / 8 / 12838 / \mathrm{P} 12838 . \mathrm{xml} \& \mathrm{xsl}=/ \mathrm{de} / \mathrm{tpl} /$ p9f.xsl\&base $=/$ tpl $/$ top-bottom.xsl Acceso en junio de 2004

4. Ruggeri Laderchi C, Saith R, Stewart F. Does it matter that we don't agree on the definition of poverty? A comparison of four approaches. Oxford: Queen Elizabeth House; 2003. (Working Paper No. 107).

5. Feres JC, Mancero X. Enfoques para la medición de la pobreza. Breve revisión de la literatura. Santiago, Chile: Comisión Económica para América Latina y el Caribe; 2001.

6. Gutiérrez-Ezpeleta E. Indicadores sociales: una breve interpretación de su estado de desarrollo. En: Sojo C, ed. Desarrollo social en América Latina: temas y desafíos para las políticas públicas. San José, Costa Rica: Facultad Latinoamericana de Ciencias Sociales; 2002.

7. Quezada C. Invisible citizen. BID America, Revista del Banco Interamericano de Desarrollo. 2001 (junio).

8. Hopenhayn M, Bello A. Discriminación étnico-racial y xenophobia en América Latina y el Caribe. Santiago, Chile: Comisión Económica para América Latina y el Caribe; 2001.

9. Torres C. Equidad y salud: desde la perspectiva de la etnicidad. Washington, D.C.: Organización Panamericana de la Salud; 2001.

10. Organización Panamericana de la Salud. Etnicidad y salud. Washington, D.C. OPS; 2003. (Documento CEE 132/16).

11. Gwatkin DR. Who would gain most from efforts to reach the Millennium De- velopment Goals for Health? An inquiry into the possibility of progress that fails to reach the poor. Health, Nutrition and Population Discussion Paper. Washington, D.C.: World Bank; 2002.

12. Franco R. Grandes temas del desarrollo social en América Latina y el Caribe. En: Sojo C, ed. Desarrollo social en América Latina: temas y desafíos para las políticas públicas. Washington, D.C.: Banco Mundial; 2002.

13. Torres C. Acciones afirmativas para lograr la equidad de salud para los grupos étnicos/raciales. Washington, D.C.: Organización Panamericana de la Salud; 2003.

14. Pan American Health Organization. Primary health care in the Americas: lessons learned over 25 years and future challenges. 44 Directing Council. Washington, D.C.: Pan American Health Organization; 2003.

15. Annan K. Discurso del Secretario General ante la XIII Cumbre Iberoamericana, Santa Cruz de la Sierra, Bolivia, 2003. Nueva York: Naciones Unidas; 2003. 